\title{
EI liderazgo del director y trabajo docente para un servicio educativo de calidad
}

\section{The principal's leadership and teaching work for a quality educational service}

DOI: $10.46932 / \mathrm{sfjdv2n2-069}$

Received in: March 1st, 2021

Accepted in: May 30th, 2021

\author{
Montalvo García Paola \\ Maestría en Administración, Gestión y Desarrollo de la Educación \\ Instituto Politécnico Nacional. ESCA Unidad Santo Tomás \\ Manuel Carpio 471, Plutarco Elías Calles, Miguel Hidalgo, 11340 Ciudad de México, CDMX \\ E-mail: paolamontalvogarcia@gmail.com \\ Olea Deserti Elia \\ Doctora en filosofía y Ciencias de la Educación \\ Instituto Politécnico Nacional. ESCA Unidad Santo Tomás \\ Manuel Carpio 471, Plutarco Elías Calles, Miguel Hidalgo, 11340 Ciudad de México, CDMX \\ E-mail: eliaolea@gmail.com
}

\section{RESUMEN}

A partir de las evaluaciones internacionales PISA, se ha identificado un problema importante en el proceso de aprendizaje en la educación básica mexicana. Se tienen las observaciones de la OCDE y el modelo educativo 2017; sin embargo, a pesar de sus bondades, consideramos se requiere atender de una manera real y desde un enfoque sistémico y de contingencia, por lo que incluimos algunas sugerencias, producto de la reflexión. Hay que otorgar relevancia a las características personales de los actores quienes participan y favorecerían el llegar a un servicio educativo innovador donde la eficacia y la eficiencia son los ejes rectores.

Palabras clave: Liderazgo del director, Factores para innovación educativa, Caracterización servicio educativo.

\begin{abstract}
From the PISA international evaluations, an important problem has been identified in the learning process in Mexican basic education. We have the observations of the OECD and the 2017 educational model; however, despite its goodness, we consider that it is required to be addressed in a real way and from a systemic and contingency approach, so we include some suggestions, product of reflection. It is necessary to give relevance to the personal characteristics of the actors who participate and would favor the achievement of an innovative educational service where effectiveness and efficiency are the guiding principles.
\end{abstract}

Key words: Principal leadership, Factors for educational innovation, Characterization of educational service. 


\section{INTRODUCCIÓN}

Hablar de los profesionales responsables de la eficacia y la eficiencia de la educación básica, fundamento de la formación y supervivencia individual y social, es hacer referencia al papel del ethos docente (directores o maestros frente a grupo). Ambos docentes son los ejes que orientan las acciones con el objetivo, que es el alcanzar el perfil del egresado de educación básica.

Desde que la Organización de las Naciones Unidades para la Educación, la Ciencia y la Cultura (UNESCO) tuvo su aparición en el devenir, ha contribuido a la paz, la erradicación de la pobreza, al desarrollo sostenible y a la educación. En su declaración de la Educación para Todos (EPT) en Jomtien Tailandia (1990) plantea la necesidad de que los niños en edad escolar, y todas las personas a nivel mundial, puedan acceder a la enseñanza básica.

De acuerdo al seguimiento de EPT se dice "cerca de 58 millones de niños en edad de cursar la enseñanza primaria (generalmente entre los seis y los once años) estaban sin escolarizar debido, entre otras razones, a las presiones demográficas, las situaciones de conflicto y la falta de la voluntad necesaria" además de la situación socioeconómica (UNESCO, 2015, p. 92); a pesar de que se ha fortalecido la equidad y la construcción de escuelas, lo que se observa ha repercutido mayormente son las becas -como transferencia en efectivo- pero sin garantizar una mejora en el desempeño escolar. Incluso en el reporte de 2016, se dice que en el mundo "Some 263 million are not in school, including 61 million children of primary school age, 60 million adolescents of lower secondary school age" (UNESCO. 2016, p. 182). Los respectivos informes de tales eventos internacionales han permitido la modificación de los planes y programas de estudio, revisado los libros de texto, formado a los docentes, elaborado material pedagógico, fomentado la participación de padres, normado el rendimiento y asignado recursos para las escuelas (UNESCO, 2015). Además, en el caso de México, se ha venido difundiendo el nuevo modelo educativo como resultado de la consulta en diversas regiones del país en el 2016, de tal forma que se estará en posibilidad de aplicarlo a partir del 2018 (SEP, 2017). Pero...este modelo por sí mismo ¿Qué sucede con el liderazgo del director de los centros escolares y la comunidad para que se cumpla con los objetivos del aprendizaje y el manejo de los recursos llegando a tener escuelas con un servicio educativo innovador?

A partir de que México se integró a la Organización para la Cooperación y el Desarrollo Económicos (OCDE) en 1994 y, de acuerdo al Instituto Nacional para la Evaluación de la Educación, en el año 2000 se inició la evaluación para estudiantes mexicanos, de 15 años, con el Programme for International Student Assessment (PISA) en donde se evalúa la calidad, equidad y eficiencia de los sistemas educativos" (OCDE, 2016, p. 2). Dicha evaluación se centra en Ciencias, Lectura y Matemáticas, en las que casi la totalidad de los alumnos del país han obtenido un resultado muy bajo. Así que, de acuerdo a los resultados de PISA 2015 se tiene que de los 70 países, México ocupa el lugar 58. En la tabla 
Tabla 1. Rendimiento obtenido por los alumnos mexicanos en el PISA 2015

\begin{tabular}{|c|c|c|c|c|}
\hline $\begin{array}{l}\text { Rendmto.en Ciencias } \\
\text { PISA } 2015 \\
\text { México }\end{array}$ & $\begin{array}{lr}\text { Rendmto. } & \text { En } \\
\text { lectura } & \text { PISA } \\
2015 & \\
\text { México } & \end{array}$ & $\begin{array}{l}\text { Rendmto. En } \\
\text { matemáticas } \\
\text { PISA 2015 } \\
\text { México }\end{array}$ & $\begin{array}{l}\% \text { alumnos de } \\
\text { nivel excelente en } \\
\text { al menos una UA } \\
\left(\begin{array}{ll}5 & \text { ó } 6)\end{array}\right.\end{array}$ & $\begin{array}{l}\% \text { alumnos con bajo } \\
\text { rendimiento en las } 3 \\
\text { UA, debajo nivel } 2\end{array}$ \\
\hline 416 & 423 & 408 & 0.6 & 33.8 \\
\hline
\end{tabular}

NOTA: UA se refiere a unidad de aprendizaje o curso. Rendmto. Es abreviatura de rendimiento.

Como se puede observar, con base en los datos de la OCDE (2016) los resultados obtenidos por los niños mexicanos en la aplicación de PISA 2015, menos del uno por ciento llega a niveles óptimos (5 ó 6) y el 34\% se ubica en el nivel 1, que es el conocimiento y aplicación básicos.

De acuerdo al informe de la OCDE, las desigualdades en los resultados en Ciencias pueden deberse a influencia de los padres de familia, docentes, políticos y líderes de opinión, más que a diferencias de género. Los directores de las escuelas mencionaron que el ausentismo del personal, el hecho de que los docentes permanecieran todos sus años de servicio en la misma escuela, con funciones iguales y un liderazgo cuyas acciones del director no propician la integración del equipo docente se consideran obstáculos para el aprendizaje de los discentes.

\section{DESARROLLO (REFLEXIÓN/APORTACIÓN)}

Hablar del liderazgo en las escuelas, es imaginar de inmediato en el director quien a través de ejercerlo, establece relaciones con la comunidad; es el responsable de la planeación, de la conducción de los currículos y así del logro de los objetivos en los ambientes de aprendizaje, de colaboración y de confianza (Araiza, Magaña y Carrillo, 2015). Para ello pone en práctica la gestión que de acuerdo con algunos autores es sinónimo de administración entendida esta última como orientada más a la organización. Si se habla de gestión educativa, se piensa en la dirección, gobierno, participación, decisiones conjuntas y evaluación (Furlán, Landermann y Pasillas, 2015), que es ejercida por el líder del centro educativo.

Ahora bien, para llegar a esa calidad educativa, hay que considerar los factores que se presentan en la figura 1 y que intervienen en el ejercicio del director escolar, de acuerdo con las autoras de esta ponencia.

Figura 1. Aspectos que influyen en un servicio educativo de calidad. 


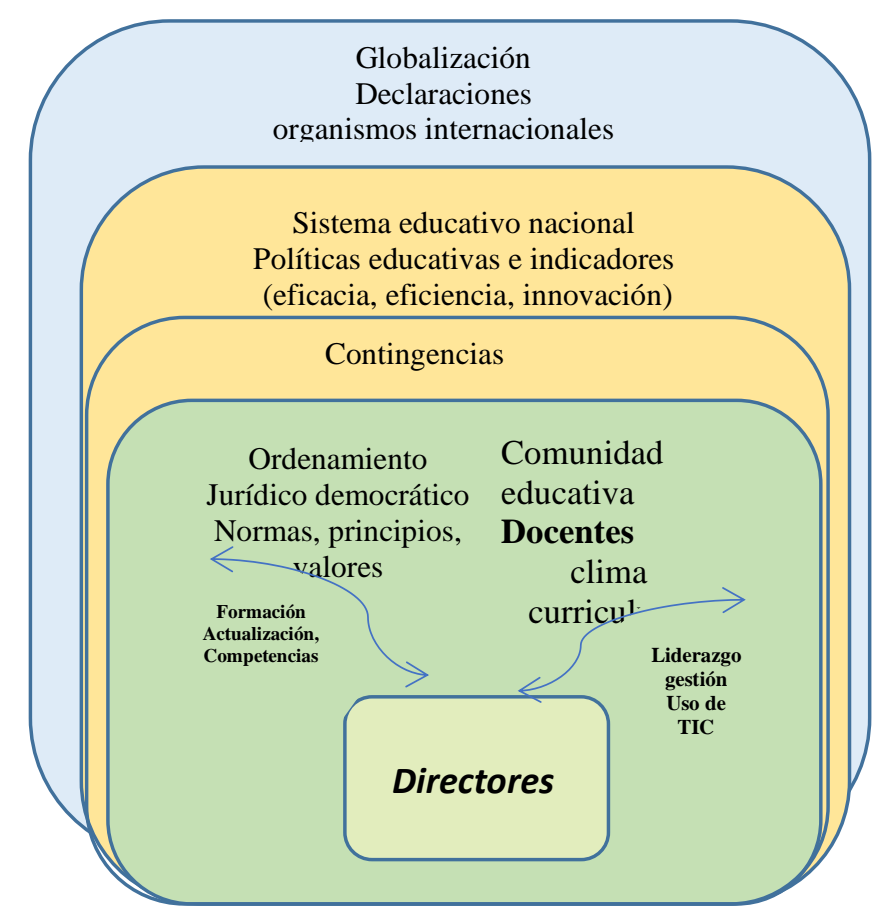

Como se puede apreciar, en estos niveles, hay influencia desde el nivel internacional y el nacional con las políticas emitidas por la Secretaría de Educación Pública, la cual va marcando los indicadores educativos. Sin embargo, un aspecto trascendente para el éxito es el considerar lo que corresponde a las teorías de sistemas y de las contingencias que surgieron a partir de los años sesenta, que explican que las organizaciones están en permanente interacción con el medio ambiente, al cual influyen y a la vez son influídas por él, por lo que adoptan ciertas estructuras y manera de laborar (Marín y Cuartas, 2014). Los factores que afectan el diseño organizacional son los que corresponden a la estrategia, el tamaño de la organización, el uso de TIC y la certeza/incertidumbre de la tarea (Donaldson, 2001, en Marín y Cuartas, 2014).

De igual forma, se puede afirmar que existe un ordenamiento jurídico que es el que influye modelando la conducta de acuerdo al medio estatutario. Sin embargo, lo más importante es la comunidad educativa que interactúa a través de sus actores en un medio ambiente determinado y con base en el(los) currículo(s); aunque son trascendentes la formación, actualización y las competencias, el uso de las TIC y el liderazgo de gestión del director.

Así que para abatir el problema que como país se tiene en cuanto al rendimiento académico de estudiantes de 15 años de edad y que fue descrita en la introducción, es conveniente considerar no sólo el modelo educativo, el tener maestros y directores preparados si se ignora lo que sucede a nivel personal en ellos mismos así como lo que sucede en el entorno que a nivel de contingencia puede alterar la dinámica de la comunidad escolar y así el trabajo planeado y establecido de acuerdo a la normativa. De ahí que en 
Tabla A. el liderazgo del director desde tres puntos de vista

\begin{tabular}{|c|c|c|}
\hline OCDE & $\begin{array}{l}\text { SEP Modelo Educativo para la } \\
\text { educación obligatoria }\end{array}$ & Montalvo y Olea \\
\hline $\begin{array}{l}\text { Selección, evaluación de líderes que } \\
\text { se ofrece y las repercusiones en el } \\
\text { estatus. }\end{array}$ & $\begin{array}{l}\text { Liderazgo directivo que permita pasar } \\
\text { de un sistema educativo vertical a uno } \\
\text { más horizontal (elaborar Ruta de } \\
\text { Mejora). La escuela es el centro de } \\
\text { gestión del sistema con recursos que } \\
\text { apoyan la organización. }\end{array}$ & $\begin{array}{l}\text { Elemento intra-escolar de gran } \\
\text { importancia para la innovación } \\
\text { (modelos, estrategias de enseñanza y } \\
\text { aprendizaje, acciones técnico } \\
\text { pedagógicas y } \\
\text { administrativas). }\end{array}$ \\
\hline $\begin{array}{l}\text { Mejora en las funciones, } \\
\text { atribuciones y estándares de los } \\
\text { directores (líderes). }\end{array}$ & $\begin{array}{l}\text { Escuelas con autonomía de gestión, } \\
\text { "plantillas de maestros y directivos } \\
\text { fortalecidos, liderazgo directivo, } \\
\text { trabajo colegiado, menor carga } \\
\text { administrativa, infraestructura digna, } \\
\text { acceso a las TIC, conectividad, } \\
\text { presupuesto propio, asistencia } \\
\text { técnico-pedagógica de calidad" (SEP, } \\
2017, \text { p. 28). Formación continua. }\end{array}$ & $\begin{array}{l}\text { Aplicar estándares que en función de } \\
\text { las metas y calidad del alcance sean } \\
\text { reflejo de las acciones, como: } \\
\text { liderazgo efectivo, clima de } \\
\text { confianza, compromiso de enseñar, } \\
\text { decisiones compartidas, planeación } \\
\text { institucional, auto-evaluación, } \\
\text { comunicación del desempeño y redes } \\
\text { escolares (Araiza, Magaña y Carrillo, } \\
\text { 2015) }\end{array}$ \\
\hline $\begin{array}{l}\text { Se acentúa más sobre los soportes } \\
\text { como la institucionalidad y la } \\
\text { propuesta política. }\end{array}$ & $\begin{array}{l}\text { Se habla del "reconocimiento de la } \\
\text { diversidad de contextos y modalidades } \\
\text { en que se desarrollan las comunidades } \\
\text { educativas" (SEP, 2017, p. 19) pero no } \\
\text { dice qué se tiene que hacer. Se habla } \\
\text { de colaboración entre el gobierno } \\
\text { federal y los estatales. }\end{array}$ & $\begin{array}{l}\text { Tiene influencia global y local y en } \\
\text { especial de la complejidad que rodea } \\
\text { el centro educativo que repercute en } \\
\text { la conducta personal y grupal de los } \\
\text { docentes y del director. }\end{array}$ \\
\hline
\end{tabular}

De hecho, las características del director se fundamentan en tres aspectos:

\subsection{EL ROL QUE DESEMPEÑA}

De acuerdo a las atribuciones concedidas es el actor principal, con mayor autoridad que se rige de acuerdo a la normativa y maneja los ámbitos de la gestión relacionadas con la organización, orientación, operación, planeación, supervisión y administración de la escuela.

\subsection{ESTÁNDARES DEL DIRECTOR}

Se basan en dos competencias funcionales: La administrativa, curricular y de recursos y las comportamentales referidas a actitudes, valores, motivaciones y dinámica de personalidad que para ejercer el liderazgo (con comunicación, relaciones interpersonales, negociación, mediación) que repercuten en un clima organizacional.

\subsection{LIDERAZGO DISTRIBUIDO}


Es compartido con otros líderes similares al director, pero de menor rango, como los subdirectores y coordinadores escolares que son encargados de las funciones técnico-pedagógicas de las escuelas y en algunos casos de las técnico-administrativas relacionadas con el control y la evaluación.

\section{LA PARTICIPACIÓN DE LOS DOCENTES}

Tanto el liderazgo como la participación son procesos claves de la autonomía, la calidad, la eficiencia, eficiencia necesarios para la calidad en el servicio que se ofrece. Al incluir la participación de la comunidad educativa surge un espacio democrático ya que se involucran activamente creando la oportunidad de generar una dinámica en la vida de la escuela (Bazo, Alfaro, Calderón y Álvarez, 2010). Referida en los niveles macro/micro, siendo este último el contexto organizacional donde se producen conflictos de manera natural y se promueve un cambio acorde a cada institución con base en metas, interés, disputas y poder, de docentes y directivos (actores) en interacción. De ahí que se deben considerar las características personales además de la normativa, plasmadas en la tabla 1.

La participación puede promover la creatividad y apoya la adaptación de los procesos de mejora continua de acuerdo a tres momentos: Discusión que origina una decisión por parte del director y los docentes; Expresión de opiniones (consultoría) y Ejecución de las decisiones tomadas por parte de los actores (Obando, 2008).

Tabla 1. Aspectos personales que influyen en la participación docente

\begin{tabular}{|l|l|}
\hline El género & $\begin{array}{l}\text { Aspectos sociales, culturales, biológicos y actitudinales que tiene tanto el hombre como la mujer en el } \\
\text { desempeño docente y deben considerarse en la implementación de estrategias. E n algunos casos puede } \\
\text { originar estrés laboral. }\end{array}$ \\
\hline Edad & Para diseñar acciones se considera los años vividos que se reflejan en madurez y experiencia. \\
\hline $\begin{array}{l}\text { A partir de su personalidad se refleja en sus actitudes y en la forma en que interactua de acuerdo a: } \\
\text { Desarrollo }\end{array}$ & $\begin{array}{l}\text { Autoestima. Expresión que hace la persona de sí misma apoyada por la creatividad, productividad } \\
\text { humano }\end{array}$ \\
& $\begin{array}{l}\text { Asertividad. Es la confianza para opinar y defender los puntos de vista. } \\
\text { Emoción. Es la interacción de sensaciones, percepciones y experiencia que despierta el ánimo de } \\
\text { participar o no en las acciones planeadas como parte del grupo docente. } \\
\text { Inteligencia. Capacidad del individuo para entender conceptos con relación a las ideas y a los } \\
\text { problemas que se presentan en su vida cotidiana, permite solucionar y sobrevivir a los problemas. }\end{array}$ \\
\hline
\end{tabular}

NOTA: Olea, Elia (2016). El tutor como figura académica para el éxito en las unidades de aprendizaje, pp. 72-76.

Por otra parte las características que tiene cada docente en particular son fundamentales en la participación de la comunidad escolar ya que esto genera una vinculación con el director, los alumnos y los padres de familia originando una escuela eficaz con una estructura y organización ejercida mediante la gestión escolar.

\section{CONCLUSIONES}


Para que se establezca la calidad en el servicio educativo, se necesita otra forma de concebir la educación en una sociedad donde la tecnología, los valores y las normas han variado afectando la conducta de los actores principales -docentes estudiantes y en especial del director- quien a través del liderazgo y su autonomía de gestión, lleva a la comunidad a la novedad, permaneciendo sin cambios de fondo. Para ir a una innovación-transformación, es necesario cambiar el sentido de la educación, lo que lleva a pensar en las decisiones técnico-pedagógicas que favorezcan la visión para así ponerlos en práctica (Aguerrondo, 2007). Por tanto, las autoras de esta ponencia consideran que hay que pensar la educación en una prospectiva de mediano plazo, en el que realmente se aplique el modelo educativo planeado para la educación obligatoria (2017) logrando articular el liderazgo con la comunidad para la toma de decisiones (gobernanza) y la utilización de las TIC (tradicionales y alternativas). Hay que responder a la sociedad del conocimiento; la organización y la gestión tienen que considerar la realidad y aprender a solucionar los problemas que se presenten, pero no se dice cómo y no se contemplan las contingencias. Para el trabajo de directores y docentes se habla de los Consejos Técnicos Escolares como "espacios de construcción colectiva y aprendizaje entre pares. En ellos, docentes y directivos, en un diálogo horizontal planean, implementan, dan seguimiento y evalúan una Ruta de Mejora” (SEP, 2017, p.100).

Aunque se dice "el modelo se adecuará a cada realidad particular... y a las posibilidades reales que presentan las escuelas y los maestros" (SEP, 2017, p. 192), pero hay que hacerlo de manera real ya que se habla del servicio de asistencia técnica a la escuela, de asesores técnico-pedagógicos, de enseñar inglés, de la estrategia transversal @ prende 2.0 y del apoyo permanente por parte de los padres de familia en cuanto aspectos socioemocionales, proceso de lectoescritura y el pensamiento lógico-matemático, que los directores deben ser la inspiración para convertir las escuelas en organizaciones para el aprendizaje.

Es recomendable no aplicar, sólo por poner en práctica, modelos educativos nuevos que sean el reflejo de orientaciones políticas, hay que fortalecer el liderazgo del director, llegar a una formación donde los docentes -y a nivel del aula, los estudiantes- vinculen la teoría con la práctica, hay que aprender a oír y ver la realidad y negociar y propiciar las interacciones en un ambiente armonioso. Hay que considerar a los actores con sus características biológicas, psicológicas, de maduración personal, laborales, contextuales. 


\section{REFERENCIAS}

Aguerrondo, I. (2007). Innovación, escuela y sistema educativo: el reto de la articulación entre niveles. En Gvirts, S. y De Podestá, M. E. Compiladoras. Mejorar la gestión directiva en la escuela. México: Granica.

Araiza, S. M., Magaña, R. y Carrill, L. E. (2015). Evaluación de la función directiva con base en estándares. Chihuahua, México: Secretaría de Educación, Cultura y Deporte.

Bazo, C. D., Alfaro, B. P. y Álvarez, M. (2010). Los protagonistas de los Consejos Educativos Institucionales: tensiones y dilemas de la participación en la gestión de las escuela pública. Revista Iberoamerica sobre Calidad, Eficacia y Cambio en Educación. Recuperado de: http://www.rinace.net/reice/numeros/arts/vol8num3/art2.pdf.

Furlán, A., Landersmann, M. y Pasillas, M.A. (2015). La gestión, pedagógica. Polémicas y casos. En Espeleta, J. y Furlán, A. Compiladores. La gestión pedagógica de la escuela. México: Correo de la UNESCO.

INEE. Bases de datos PISA. Recuperado de: http://www.inee.edu.mx/index.php/bases-de-datos /basesde-datos-pisa.

Marín, D. A., Cuartas, J.C. Teorías del análisis y diseño organizacional: una revisión a los postulados contingentes y de la co-alineación estratégica en Revista Facultad de Ciencias Económicas: Investigación y Reflexión, 2014, XXII (Enero-Junio) Recuperado de: http://www.redalyc.org/articulo.oa?id=90931814011

Obando, C. G. (2008). La participación docente en la toma de decisiones desde la visión micropolítica. Revista Educación. Recuperado de: http:// revistas. pucp.edu. pe/index.php/ educacion/article/view/1804 OCDE. (2010). Mejorar la escuelas. Estrategias para la acción en México. Recuperado de http://www.dgespe.sep.gob.mx/public/gt-en/8-nov-2010/Mejorar\%20escuelas\%20estrategias\%

20M\%C3\%A9xico.pdf.

OCDE. (2016). Pisa 2015. Resultados Clave. Recuperado de: https://www.oecd.org/pisa/pisa-2015results-in-focus-ESP.pdf.

Olea, E. (2016). El tutor como figura académica para el éxito en las unidades de aprendizaje. México: IPN

OREALC. (2014). El liderazgo Escolar en América Latina y el Caribe. Un estado del arte con base en ocho sistemas escolares de la región. Recuperado de: http://unesdoc.unesco.org/images/

0023/002327/232799s.pdf.

SEP. (2017). Modelo Educativo para la educación obligatoria. México: SEP.

UNESCO. (s/f). Instrumentos normativos. Recuperado de http://www.unesco.org/new/es/ education/standards-and-norms.

Declaración Mundial sobre Educación para Todos y Marco de Acción para Satisfacer las Necesidades Básicas de Aprendizaje. Jomtien, Tailandia, 5-9 marzo 1990. Recuperado de http://www.unesco.org/education/pdf/JOMTIE_S.PDF/. 
(2015). La educación para todos 2000-2015: logros y desafíos. Recuperado de: http://unesdoc.unesco.org/images/0023/002324/232435s.pdf.

(2016). Education for people and planet: Creating Sustainable Futures for all. Global Education Monitoring Report. Recuperado de http://unesdoc.unesco.org/images/0024/ 002457/245752e.pdf. 